\title{
Simulation of three-dimensional rapid free-surface granular flow past different types of obstructions using the SPH method
}

\author{
AHMED M. ABDELRAZEK, ${ }^{1,2}$ ICHIRO KIMURA, ${ }^{1}$ YASUYUKI SHIMIZU ${ }^{1}$ \\ ${ }^{1}$ Hydraulic Research Laboratory, Graduate School of Engineering, Hokkaido University, Hokkaido, Japan \\ ${ }^{2}$ Irrigation Eng. \& Hydraulic Department, Faculty of Engineering, Alexandria University, Alexandria, Egypt \\ Correspondence: A. M. Abdelrazek <ahmedmrazek@yahoo.com>
}

\begin{abstract}
In nature, when hazardous geophysical granular flows (e.g. a snow avalanche) impact on an obstacle as they stream down a slope, rapid changes in flow depth, direction and velocity will occur. It is important to understand how granular material flows around such obstacles in order to enhance the design of defense structures. In this study, a three dimensional (3-D) Smoothed Particle Hydrodynamics (SPH) model is developed to simulate granular flow past different types of obstacles. The elastic-perfectly plastic model with implementation of the Mohr-Coulomb failure criterion is applied to simulate the material behavior, which describes the stress states of soil in the plastic flow regime. The model was validated by simulating the collapse of a 3-D column of sand with two different aspect ratios; the results showed that the SPH method is capable of simulating granular flow. The model is then applied to simulate the gravity-driven granular flow down an inclined surface obstructed by a group of columns with different spacing, a circular cylinder and a tetrahedral wedge. The numerical results are then compared with experimental results and two different numerical solutions. The good agreements obtained from these comparisons demonstrate that the SPH method may be a powerful method for simulating granular flow and can be extended to design protective structures.
\end{abstract}

KEYWORDS: elastic-plastic material, granular flow, particle methods, shock wave, SPH method

\section{INTRODUCTION}

Natural granular flows such as soil liquefaction, debris flows and snow avalanches cause damage to property and lead to loss of human life (Armstrong and others, 1992). Such types of flows frequently impact on obstructions as they stream down an inclined surface, producing fast changes in flow height and velocity in their neighbouring region (e.g. Savage, 1979; Tai and others, 2001; Gray and others, 2003; Hakonardottir and Hogg, 2005; Cui and others, 2007; Gray and Cui, 2007; Vreman and others, 2007; Jóhannesson and others, 2009; Johnson and Gray, 2011). Studying granular materials and their mechanism of deformation around such obstacles is very important for designing defenses and dissipating structures (e.g. Sigurdsson and others, 1998; Tai and others, 1999; Cui and others, 2007; Hauksson and others, 2007; Jóhannesson and others, 2009), such as straight or curved walls, pyramidal (tetrahedral) and cylindrical-shaped structures, etc.

The simulation of granular flow often involves modeling large motions of discrete particles, as well as modeling the deformation of the soil mass. In previous studies, many methods such as the discrete element method (Silbert and others, 2001; Faug and others, 2009), the Finite-Element Method (Kabir and others, 2008) and the depth-averaged shallow-water type theories that have additional momentum source terms (e.g. Grigourian and others, 1967; Savage and Hutter, 1989; Koch and others, 1994; Iverson, 1997; Gray and others, 1999, 2003; Mangeney-C and others, 2003) have been utilized to solve problems dealing with granular materials. However, using the Discrete Element Method is limited to small-scale problems because it is computationally demanding. Moreover, the Finite-Element Method cannot handle problems with large deformations where mesh distortion may occur.

Recently, a new class of numerical methods, which are called mesh-free methods, has been developed. Mesh-free methods do not require Eulerian grids and they deal with a number of particles in a Lagrangian framework. They are considered more effective than the Eulerian methods in dealing with large-scale problems involving large deformations. The key idea of these methods is to provide an accurate and a stable numerical solution of partial differential equations using a set of distributed particles in the physical domain without using any grids.

Many mesh-free methods have been developed in the past few decades, such as the Moving Particles Semi-implicit Method (MPS), the Diffuse Element Method (DEM), the Element-Free Galerkin Method (EFG/EFGM), the Finite Pointset Method (FPM), etc. Among these methods, the Smoothed Particle Hydrodynamics (SPH) method is widely used. SPH is a mesh-free Lagrangian method developed by Lucy, (1977); Gingold and Monaghan, (1977) in order to solve astrophysical problems in three-dimensional (3-D) open space. It has been successfully applied to a huge range of applications such as the dynamic response of material strength (Libersky and Petschek, 1991), free surface fluid flow (Monaghan, 1994; Abdelrazek and others, 2014a), incompressible fluid flow (Cummins and Rudman, 1999), multi-phase flow (Monaghan and Kocharyan, 1995), turbulent flow (Monaghan, 2002) and snow avalanches (Abdelrazek and others, 2014b, c). In the SPH method, each particle in the domain conveys all fields variable data 
such as density, pressure and velocity, and it moves with the material velocity. The governing equations in the form of partial differential equations are converted to the particle equations of motion and then they are solved using a suitable numerical scheme (the Predictor-Corrector algorithm). In the present study, the elastic-perfectly plastic model based on Mohr-Coulomb's failure criterion is implemented in SPH formulations to model the granular movement.

The motivation of this paper is to test the predictive power of the SPH method to simulate 3-D granular flows past different types of obstacles that involve large deformations. First of all, the model is validated by simulating the collapse of 3-D axisymmetric sand columns with two aspect ratios to check the applicability of SPH in simulating the granular flow. The results showed a good agreement with experimental results in terms of the final runoff distance and the deposition shape.

Secondly, the model is applied to simulate the gravity granular flow down an inclined plane obstructed by three different types of obstacles. The numerical results from these three cases are then compared with the experimental data. They are also compared with the hydraulic avalanche model solution for the second case, and the Savage and Hutter theory solution for the third case. The results obtained from this paper indicate a good agreement in terms of the formation of shock waves, dead zones and granular vacuums, and show that SPH could be a powerful method for simulating granular flows. In the following sections, the governing equations in the SPH method are discussed, as well as the implementation of soil constitutive models in the SPH framework. The results of the calculations are then introduced and discussed.

\section{GOVERNING EQUATIONS}

The governing equations for dry soil in the framework of SPH consist of linear momentum and continuity equations expressed:

$$
\begin{gathered}
\frac{D \rho}{D t}=-\rho \frac{\partial \nu^{\alpha}}{\partial x^{\alpha}}, \\
\frac{D \nu^{\alpha}}{D t}=\frac{1}{\rho}\left(\frac{\partial \sigma^{\alpha \beta}}{\partial x^{\alpha}}\right)+f^{\alpha},
\end{gathered}
$$

where $\alpha$ and $\beta$ denote the Cartesian components $x, y$ and $z$ with the Einstein convention applied to repeated indices, $\rho$ is the density, $v$ is the velocity, $\sigma^{\alpha \beta}$ is the stress tensor, $f^{\alpha}$ is the component of acceleration caused by external force, and $D / D t$ is the material derivative, which is defined as

$$
\frac{D}{D t}=\frac{\partial}{\partial t}+v^{\alpha} \frac{\partial}{\partial x^{\alpha}} .
$$

Usually the stress tensor, $\sigma^{\alpha \beta}$, consists of two parts: an isotropic pressure $P$ and a deviatoric stress $S$;

$$
\sigma^{\alpha \beta}=-P \delta^{\alpha \beta}+S^{\alpha \beta}
$$

\section{CONSTITUTIVE EQUATIONS FOR SOIL}

Modeling the behavior of dry soil using the SPH method is similar to that for water, in which the soil is presumed to behave as a quasi-compressible material. The soil is approximated by an artificial material that is more compressible than the real one- the artificial compressibility technique (Liu and Liu, 2003). The key difference between these two models is in the calculation of the stress tensor, in which the pressure and stress/strain relationship for soil are calculated differently. Following Bui and others (2007) herein, the soil is assumed to be an elastic-plastic material. The pressure term in Eqn (4) is normally calculated using an 'equation of state', which has a function of density change (one of the fundamentals of the SPH method); thus, the pressure equation of soil will obey Hooke's law (Bui and others, 2007):

$$
P=-K \frac{\Delta V}{V}=K\left(\frac{\rho}{\rho_{\mathrm{o}}}-1\right)
$$

where $K$ is the bulk modulus, $\Delta V / V$ is the volumetric strain, and $\rho_{\mathrm{O}}$ is the initial density of the soil. Using the real value of $K$ a stiff behavior of soil will be obtained. Therefore, $K$ should be chosen as small as possible in order to ensure a near incompressibility condition and to avoid the stiff behavior (minimizing pressure fluctuations). In this study, we chose $K=50 \rho_{\mathrm{o}} g H_{\max }$ In Eqn (5) (50 times the maximum initial pressure).

Since the soil is assumed to possess elastic behavior (Bui and others, 2007, 2008, 2010; Yaidel and others, 2013), the rate of change of deviatoric shear stress, $d S / d t$, can be calculated using shear modulus, $\mu$, using the Jaumann rate from the following constitutive equation:

$$
\frac{d S^{\alpha \beta}}{d t}=2 \mu\left(\dot{\boldsymbol{\varepsilon}}^{\alpha \beta}-\frac{1}{3} \dot{\boldsymbol{\varepsilon}}^{\gamma \gamma}\right)+S^{\alpha \gamma} \omega^{\beta \gamma}+\omega^{\gamma \beta} S^{\alpha \gamma},
$$

where $\dot{\boldsymbol{\varepsilon}}^{\gamma \gamma}=\dot{\boldsymbol{\varepsilon}}^{x x}+\dot{\boldsymbol{\varepsilon}}^{y y}+\dot{\boldsymbol{\varepsilon}}^{z z}, \dot{\varepsilon}$ is the strain rate tensor and $\omega$ is the rotation rate tensor. They can be defined as:

$$
\begin{aligned}
\dot{\varepsilon}^{\alpha \beta} & =\frac{1}{2}\left(\nabla \nu^{\alpha \beta}+\nabla \nu^{\beta \alpha}\right), \\
\omega^{\alpha \beta} & =\frac{1}{2}\left(\nabla \nu^{\alpha \beta}-\nabla \nu^{\beta \alpha}\right),
\end{aligned}
$$

where $\nabla v^{\alpha \beta}=\partial \nu^{\alpha} / \partial x^{\beta}$, is the velocity gradient.

\section{SMOOTHED PARTICLE HYDRODYNAMICS (SPH) FORMULATION}

\subsection{SPH concepts and the discretization of governing equations}

The SPH method is a continuum-scale numerical method. The material properties, $f(x)$, at any point $x$ in the simulation domain are calculated according to an interpolation theory over those neighboring particles within its influence domain, $\Omega$, as shown in Figure 1 (Gingold and Monaghan, 1977; Lucy, 1977; Monaghan and Kocharyan, 1995; Liu and Liu, 2003; Hanifa and others, 2013), through the following formula:

$$
f(x)=\int_{\Omega} f\left(x^{\prime}\right) W\left(x-x^{\prime}, h\right) d x^{\prime},
$$

where $h$ is the smoothing length defining the influence domain of the kernel estimate and $W\left(x-x^{\prime}, h\right)$ is the smoothing function, which must satisfy three conditions: the 


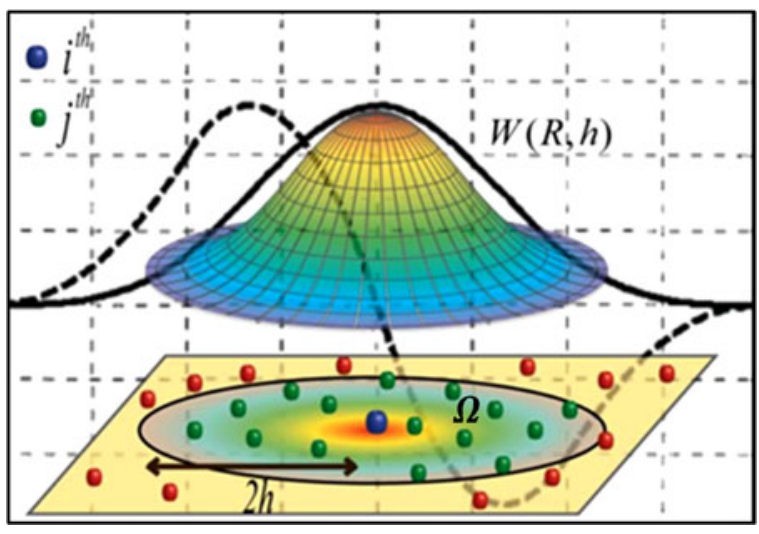

Fig. 1. Particle approximation based on kernel function $W$ in influence domain $\Omega$ with radius $k h(k=2)$.

normalization condition, delta function property and the compact condition (Liu and Liu, 2003).

There are many possible types of smoothing functions that can satisfy the aforementioned conditions. One of the most known functions among them is the cubic spline interpolation function, which was proposed by Monaghan and Lattanzio (1985), and it is defined as:

$$
W(R, h)=\alpha_{\mathrm{d}} \times\left\{\begin{array}{lc}
1.5-R^{2}+0.5 R^{3} & 0 \leq R<1, \\
(2-R)^{3} / 6 & 1 \leq R<2, \\
0 & R \geq 2,
\end{array}\right.
$$

where $\alpha_{\mathrm{d}}=3 / 2 \pi h^{3}$ in 3-D space and $R=\left(x-x^{\prime}\right) / h$.

Using the SPH approximation, which is discussed and summarized in many references (Liu and Liu, 2003, 2010), the system of partial differential Eqs (1) and (2) can be converted into the SPH formulations, which are used to solve the motion of the particles representing the soil as follows:

$$
\begin{gathered}
\frac{D \rho_{i}}{D t}=\sum_{j=1}^{N} m_{j}\left(v_{i}^{\alpha}-v_{j}^{\alpha}\right) \frac{\partial W_{i j}}{\partial x_{i}^{\alpha}}, \\
\frac{D v_{i}^{\alpha}}{D t}=\sum_{j=1}^{N} m_{j}\left(\frac{\sigma_{i}^{\alpha \beta}}{\rho_{i}^{2}}+\frac{\sigma_{j}^{\alpha \beta}}{\rho_{j}^{2}}\right) \frac{\partial W_{i j}}{\partial x_{i}^{\beta}}+f^{\alpha} .
\end{gathered}
$$

Similarly, the SPH approximation of the velocity gradient for particle $i$ can be expressed as follows:

$$
\nabla v_{i}^{\alpha \beta}=\sum_{j=1}^{N} \frac{m_{j}}{\rho_{j}}\left(v_{i}^{\alpha}-v_{j}^{\alpha}\right) \frac{\partial W_{i j}}{\partial x_{i}^{\beta}} .
$$

Therefore, the strain rate tensor and the rotation rate tensor for particle $i$ can be calculated as follows:

$$
\begin{aligned}
\dot{\varepsilon}_{i}^{\alpha \beta} & =\frac{1}{2}\left(\nabla v_{i}^{\alpha \beta}+\nabla \nu_{i}^{\beta \alpha}\right), \\
\omega_{i}^{\alpha \beta} & =\frac{1}{2}\left(\nabla \nu_{i}^{\alpha \beta}-\nabla v_{i}^{\beta \alpha}\right),
\end{aligned}
$$

By combining Eqs (6), (14) and (15), the deviatoric shear stress components can be calculated.

The plastic flow regime is determined by the MohrCoulomb failure criterion and the deviatoric shear stress components of soil in this region are scaled back to the maximum shear stress defined by $\left(S_{\mathrm{f}}=c+P \tan \phi\right)$. Here, $c$ is the soil cohesion and $\phi$ is the angle of internal friction of the soil. The above soil constitutive model used three soil parameters: cohesion (c), internal friction $(\phi)$ and shear strength $(\mu)$.

At this stage, we can calculate the pressure and the deviatoric shear stress components for every particle in the domain, and by using the Predictor-Corrector algorithm the field variables, which include velocity $(v)$, density $(\rho)$, stress tensor $\left(\sigma^{\alpha \beta}\right)$ and particle positions are updated at each subsequent time step.

\subsection{Artificial viscosity}

In order to damp out the unphysical stress fluctuation, prevent shock waves and prevent unphysical penetration for particles approaching each other, an artificial viscosity $\left(\pi_{i j}\right)$ has been introduced to the pressure term in the momentum equation. The most widely used type is proposed by Monaghan and Lattanzio (1985), and specified as:

$$
\pi_{i j}= \begin{cases}\frac{-\alpha_{1} \bar{c}_{i j} \phi_{i j}+\beta_{1} \phi_{i j}^{2}}{\bar{\rho}_{i j}} & v_{i j} . x_{i j}<0, \\ 0 & v_{i j} . x_{i j} \geq 0,\end{cases}
$$

where

$$
\begin{gathered}
\phi_{i j}=\frac{\bar{h}_{i j} v_{i j} \cdot x_{i j}}{\left|x_{i j}\right|^{2}+\varepsilon \bar{h}_{i j}^{2}}, \bar{c}_{i j}=\frac{1}{2}\left(c_{i}+c_{j}\right), \\
\bar{h}_{i j}=\frac{1}{2}\left(h_{i}+h_{j}\right), \bar{\rho}_{i j}=\frac{1}{2}\left(\rho_{i}+\rho_{j}\right), \\
v_{i j}=v_{i}-v_{j}, x_{i j}=x_{i}-x_{j},
\end{gathered}
$$

where $\alpha_{1}$ and $\beta_{1}$ are constants and both given the value of $1.0, \varepsilon$ is usually taken as $0.01, c$ is the speed of sound and $v$ represents the particle velocity vector.

Ultimately, the momentum equation after introducing the artificial viscosity is in the form:

$$
\frac{D v_{i}^{\alpha}}{D t}=\sum_{j=1}^{N} m_{j}\left(\frac{\sigma_{i}^{\alpha \beta}}{\rho_{i}^{2}}+\frac{\sigma_{j}^{\alpha \beta}}{\rho_{j}^{2}}+\pi_{i j}\right) \frac{\partial W_{i j}}{\partial x_{i}^{\beta}}+f^{\alpha} .
$$

\subsection{Boundary conditions}

In this study, a dynamic boundary condition is used to represent boundary particles, which are forced to follow the governing equations (continuity, momentum and state equations), but they are fixed. When the particles move closer toward the boundary, the density of the boundary particles increases, according to the continuity equation, which leads to an increase in the pressure following the equation of state. Subsequently, the force exerted on the approaching particles increases because of the pressure term in the momentum equation by the generations of repulsion between the material particles and the boundary particles (Dalrymple and Knio, 2001). The boundary particles are set in a staggered manner in order to prevent particle leakage as shown in Figure 2. Treatment of the frictional boundary condition is not implemented in this study. Improvement is 


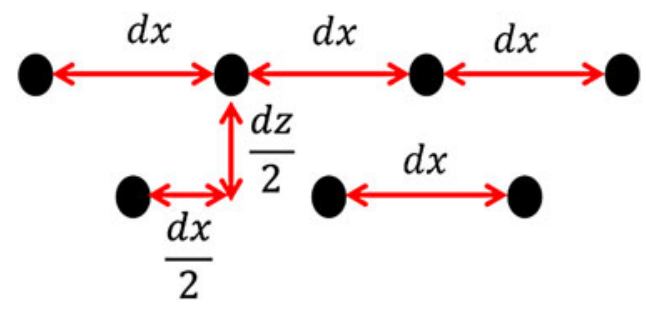

Fig. 2. Arrangement of boundary particles.

needed in the boundary condition in order to treat the granular mass as a frictional Coulomb-like continuum.

\section{TEST CASE}

In order to validate our model and show its capability, numerical simulations were conducted to simulate the experiments done by Lube and others (2004) on the collapse of a vertical, 3-D, axisymmetric column of sand. Lube and others (2004) pointed out that the flow behavior of granular columns relies on the aspect ratio $a=H_{\mathrm{i}} / R_{\mathrm{i}}$, where $H_{\mathrm{i}}$ and $R_{\mathrm{i}}$ are the initial height and radius of the granular column, respectively. They concluded that the final runoff distance and collapsed column height can be expressed only in terms of aspect ratio and the initial radius, and is independent of any friction coefficient. Two experiments were selected with aspect ratios of 0.9 and 2.75 , and simulated using the $\mathrm{SPH}$ model. The initial radius of the column was $0.1 \mathrm{~m}$ and the numbers of fluid particles used in these simulations were 22590 and 69025 , with $0.005 \mathrm{~m}$ initial distance between particles. Values of Young's modulus, the Poisson ratio, friction angle and density were $5 \mathrm{MPa}, 0.3,30^{\circ}$ and $2600 \mathrm{~kg} \mathrm{~m}^{-3}$, respectively.

Figure 3 shows a comparison between the numerical and experimental results for the collapse of the sand column with $a=0.9$. The numerical results show good agreement with the experimental results in terms of the final runout distance and deposit profile. However, we have indicated differences in terms of the final top shape of the cone and its sharpness. Lube and others (2004) indicate that a very sharp cone at the top of the final cone remains at the initial height, but in the SPH simulation, a slight decrease in the final height is observed as shown in Figure 3c. This is probably because the number of particles is not adequate to give smooth and reliable results or because of the truncation error that resulted from the approximation of spatial derivatives. Many modifications and improvements have been proposed to the $\mathrm{SPH}$ method to overcome these types of errors (Amicarelli and others, 2011; Hopkins, 2014), but they are not considered in this paper. Moreover, a zero dilation angle of sand is assumed for simplicity, which led to a weaker soil in the SPH model (Chen and Qiu, 2012). In addition, the angle of repose, after the soil collapse in the simulation, is slightly smaller than that obtained from the experimental results.

Figure 4 represents a comparison between experimental and numerical results in terms of the radius of the flow front as functions of time for the case with an aspect ratio of 0.9. Even though there are small differences between the $\mathrm{SPH}$ and experimental results, the model is in a good quantitative agreement with the experiment, both in the position of the radius of the flow front and the timescale for their
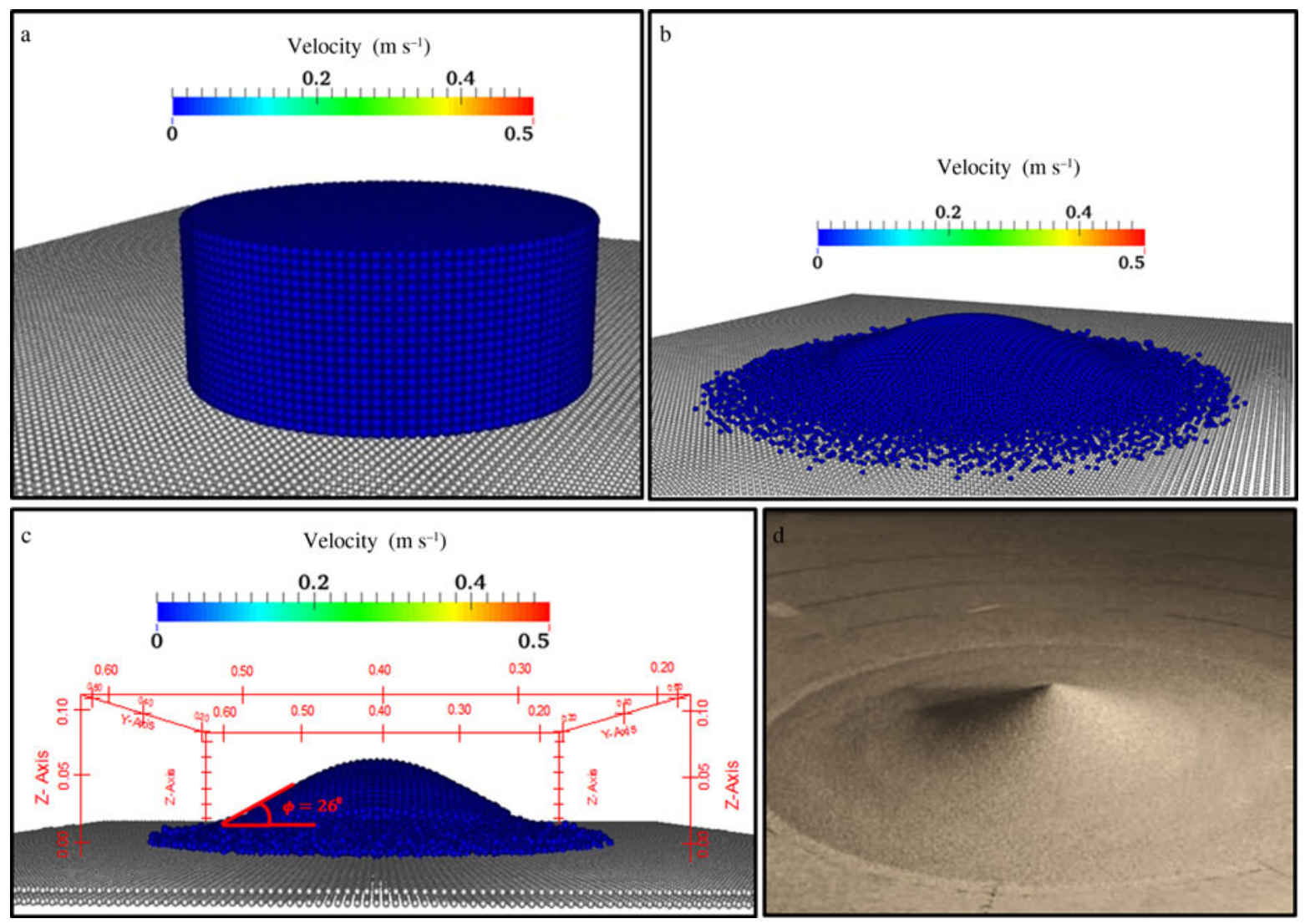

Fig. 3. Comparison between experimental and numerical results: (a) initial shape of sand column; (b) simulated final profile; (c) side view of the simulated final profile; (d) experimental final profile (Lube and others (2004)). 


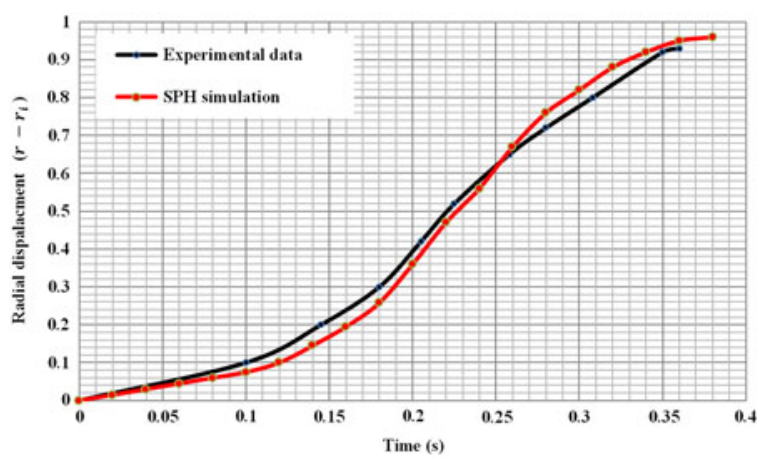

Fig. 4. Typical radial displacements as functions of time for the case with an aspect ratio equal to 0.9 .

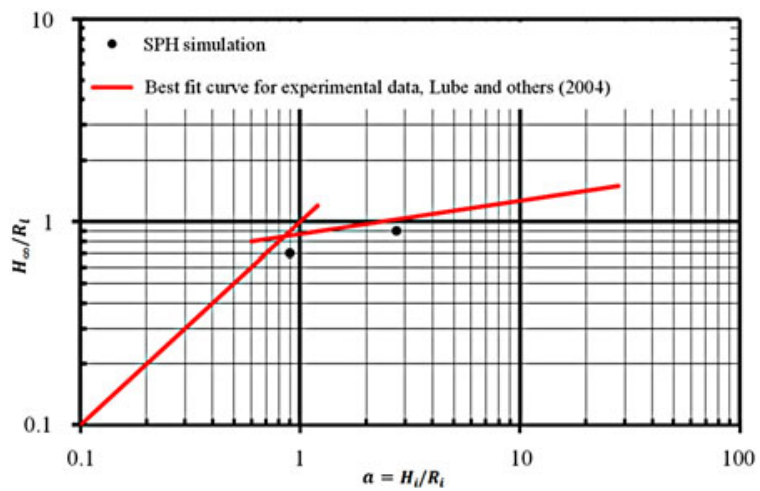

Fig. 5. Comparison of normalized final deposit height between the simulations and experimental results.

formation. Also, the flow patterns in both experiment and simulation have a primary acceleration phase followed by a deceleration phase.

Figure 5 shows a comparison of the normalized final deposit height of the SPH simulations in the two cases, together with a fit curve from the experimental data observed by Lube and others (2004). It illustrates that the computed final deposit height is slightly lower than the values predicted from the experimental data.

\section{RESULTS AND DISCUSSIONS}

In this section, $\mathrm{SPH}$ is applied to simulate Geotechnical materials through three main problems to test the abilities of the proposed model. First, SPH is applied to simulate a small-scale granular avalanche flow down an inclined surface obstructed by a group of stake rows with different spacing. Through this case study, we show that SPH can capture the deposition shape at the end of the slope. Next, the SPH model is applied to simulate the 3-D granular freesurface flow around a circular cylinder which considers common obstacles such as tree trunks, and is of fundamental practical interest to the design of pylons that are able to show steadfastness in front of such kinds of flows (Sovilla and others, 2008).

Finally, the SPH method is used to carry out 3-D simulations of the gravity granular flow past a pyramid wedge (tetrahedral) which is used as a typical defense structure to divert flow and guide avalanches past protected buildings and other areas.

\subsection{Gravity granular flows past a group of stake rows}

\subsubsection{Model description}

A laboratory experiment was conducted by Yellin and others (2013) in the Hydraulics Research Laboratory at Hokkaido University. Figure 6 shows a schematic diagram illustrating the dimensions of the experiment and the initial position of a sand mass. The model was built from two wooden panels; each of them is $1.80 \mathrm{~m}$ long and $0.90 \mathrm{~m}$ wide. The first panel was inclined at $20^{\circ}$ with the horizontal and the obstacles were placed at a distance of $30 \mathrm{~cm}$ from its top. The inclination of the second panel was $45^{\circ}$. An amount of quartz sand $(2.04 \mathrm{~kg})$ was initially filled in the starting box, located $30 \mathrm{~cm}$ from the top of the second panel. Grid lines were drawn on the panels, with $10 \mathrm{~cm}$ intervals in order to know the particle positions and their speed.

This experiment was originally conducted as a starting point to design energy dissipators for the snow avalanche. We used three different types of obstacles, as shown in Figure 7 .

Case 1: $1 \mathrm{~cm}$ square columns with $1 \mathrm{~cm}$ spacing. Case 2: $2 \mathrm{~cm}$ square columns with $2 \mathrm{~cm}$ spacing. Case 3: $1 \mathrm{~cm}$ square columns in a staggered shape.
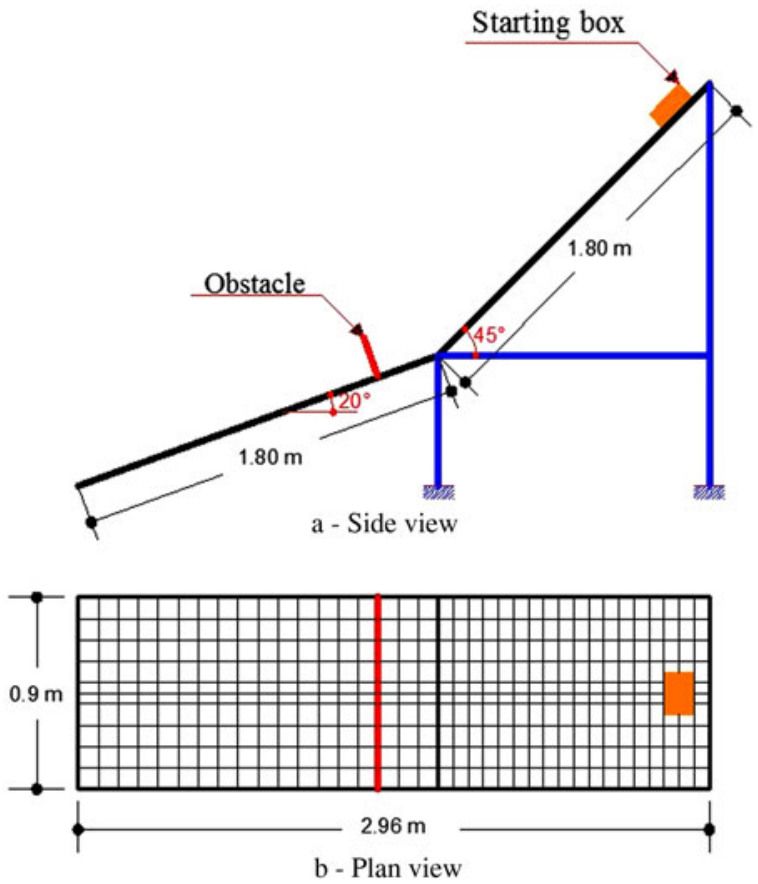

Fig. 6. Schematic sketch of the avalanche experiment.

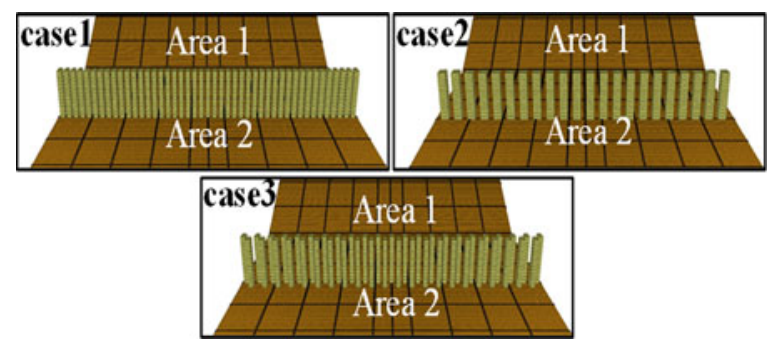

Fig. 7. The different types of obstacles, and areas name. 


\subsubsection{Numerical simulations}

The simulation of the granular flow was carried out using the proposed SPH model. The number of particles used to simulate the soil mass was 12054 , with $0.005 \mathrm{~m}$ initial distance between particles. These particles had the following properties: density $\rho=1500 \mathrm{~kg} \mathrm{~m}^{-3}$, Young's modulus $E=150 \mathrm{MPa}$, internal friction angle $\phi=32^{\circ}$ and Poisson ratio $v=0.3$

\subsubsection{Results and analysis}

Figures 8-10 show the comparisons between the experimental and numerical results in terms of the final deposition shape and particles spreading at the three cases. A good agreement between the simulated and experimental results is found when we use a suitable number of particles. However, we notice that the final simulated deposition shapes are almost symmetrical around the longitudinal centerline, unlike the deposition forms resulting from the experiments. Also, in Cases 1 and 3, some particles were deposited above the obstacle in the experimental results while in Case 2, with the wider spacing between obstacles, there is no deposition above the obstacle. The results are successfully confirmed by the numerical results. Figure 11 represents a comparison between the experimental and numerical results in terms of the position of the leading edge vs time for Case 1. Although there are small differences between the $\mathrm{SPH}$ and experimental results, the positions of the leading edge stay almost the same in both the numerical and experimental results until $t=1.4 \mathrm{~s}$. After that, the speed of the particles in the experiment decreased, and finally stopped at $t=3.0 \mathrm{~s}$, while the simulated particles moved faster until $t=1.7 \mathrm{~s}$, then gradually stopped at $t=3.2 \mathrm{~s}$
In order to investigate the efficiency of the obstacles, Yellin and others (2013) designated the area upstream of the obstacles as Area 1, and the area downstream Area 2 , as shown in Figure 7 , and then they measured the weight of deposited material (A1 and $\mathrm{A} 2$ respectively) in each area and derived an obstacle efficiency factor: Efficiency factor $(\%)=(\mathrm{A} 1+\mathrm{A} 2) / 2.04 \mathrm{~kg} \times 100 \%$. In our simulations, we counted the number of deposited particles in both the areas. Table 1 shows the efficiency factor for the experimental and simulated results. The efficiency factor from the experimental results in all cases is slightly greater than that calculated from the SPH results; the error is about $5 \%$.

\subsection{Granular free-surface flow around a circular cylinder}

\subsubsection{Model description}

Small-scale experiments were carried out by Cui and others (2013) to investigate the gravity-driven free-surface flow of a granular avalanche around a circular cylinder.

The experimental device was built using a smooth Plexiglas chute that is $300 \mathrm{~mm}$ wide and $600 \mathrm{~mm}$ long and is inclined at an angle $\zeta=36^{\circ}$ to the horizontal as shown in Figure 12. Sand was loaded into a hopper at the top of the chute, and a gate was used to control the height and flux of material entering the chute. A $30 \mathrm{~mm}$ diameter circular metal cylinder was attached to the center of the chute $300 \mathrm{~mm}$ downstream from the inflow gate, so that its axis was normal to the inclined plane.

All phases of the experiments were totally registered with a video and photo camera. Pictures were taken at a rate of $25 \mathrm{~s}^{-1}$ in order to show how the bow shock and
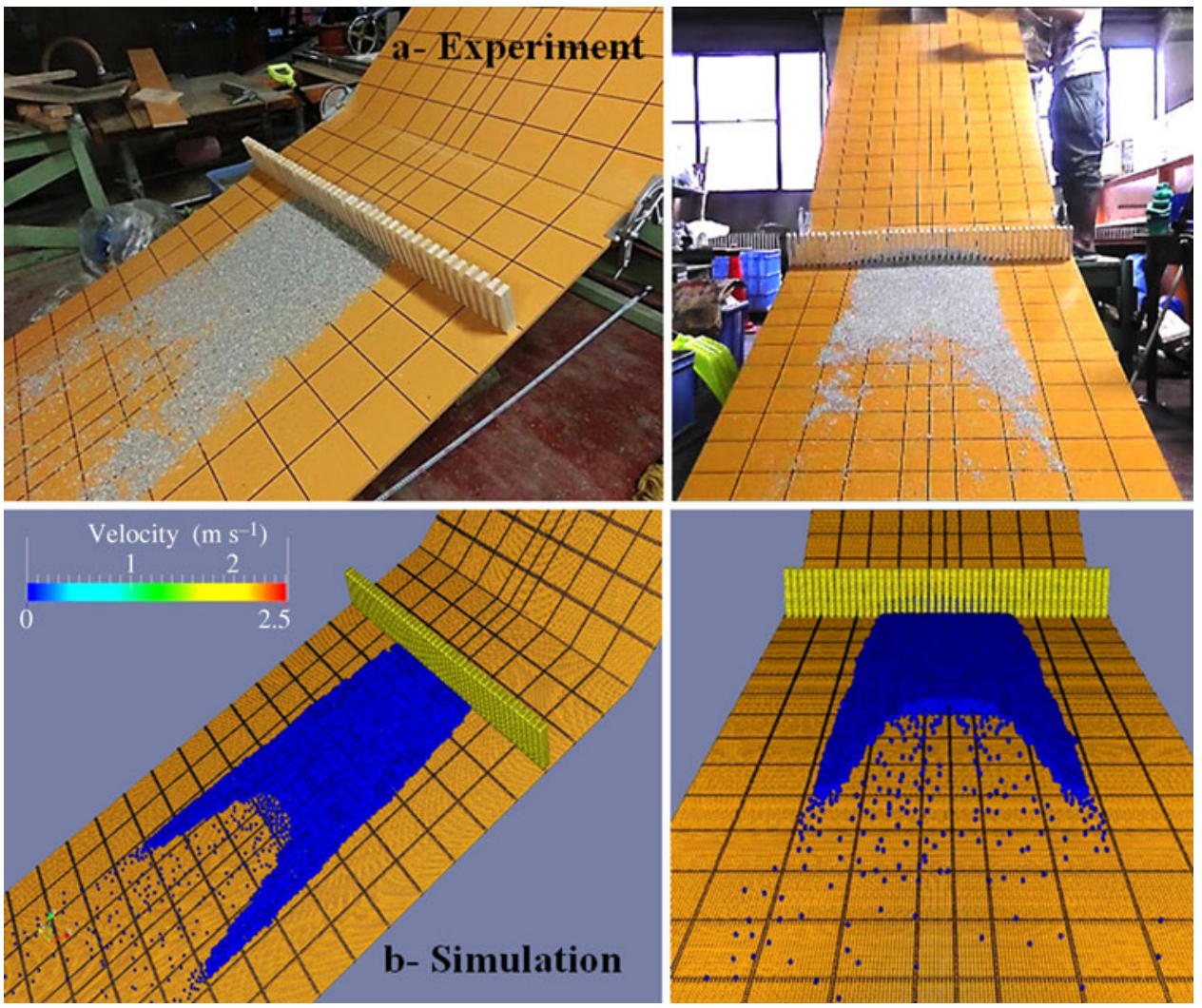

Fig. 8. Comparison between: (a) experimental and (b) numerical results in term of the final deposition shape (Case 1). 

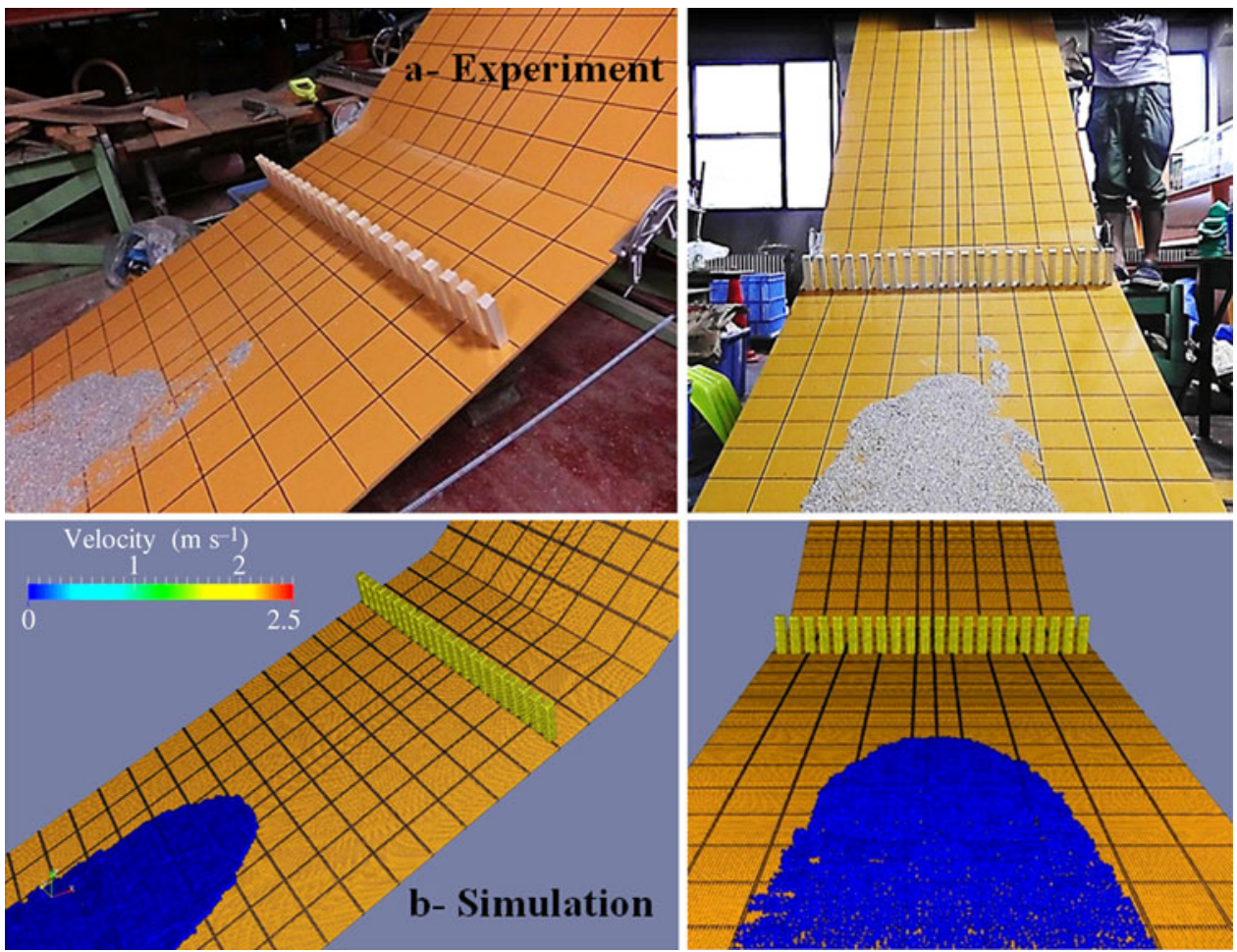

Fig. 9. Comparison between: (a) experimental and (b) numerical results in term of the final deposition shape (Case 2).
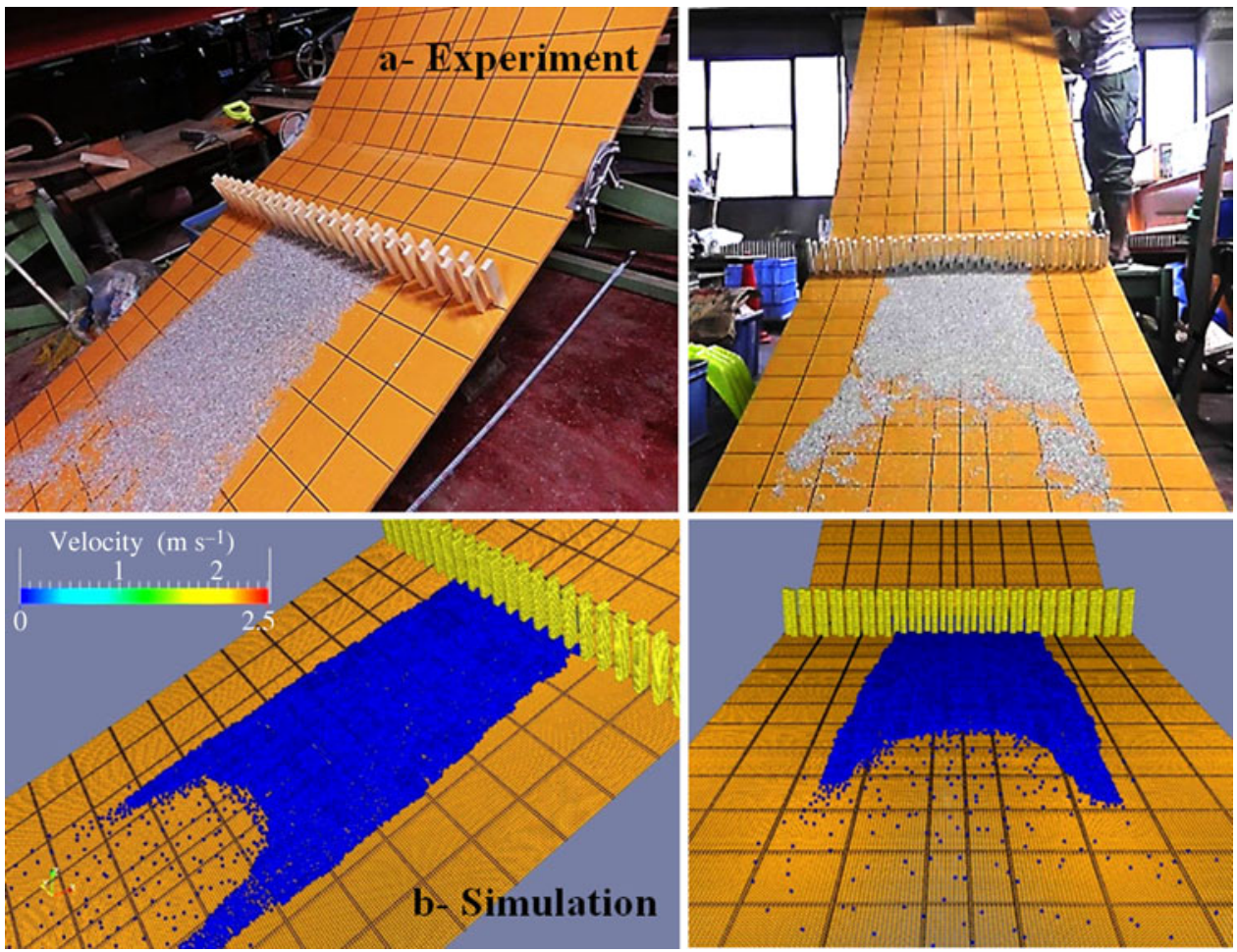

Fig. 10. Comparison between: (a) experimental and (b) numerical results in term of the final deposition shape (Case 3).

the granular vacuum are formed in a close-up region near the cylinder.

\subsubsection{Numerical simulations}

In total, 234675 soil particles with an initial mean separation of $0.002 \mathrm{~m}$ were used to simulate a sand mass. They were placed in the hopper at the top of the chute. Table 2 shows the values of the parameters used to simulate the flow of a granular avalanche around a circular cylinder.

The gate opening height is 0.4 dimensionless units, where the cylinder diameter $D=30 \mathrm{~mm}$ is used in dimension scaling (30 $\mathrm{mm}=1$ dimensionless unit). On the other hand, the velocities are scaled by $\sqrt{L g}=0.54 \mathrm{~ms}^{-1}$ and time is scaled by $\sqrt{L / g}=0.055 \mathrm{~s}$. 


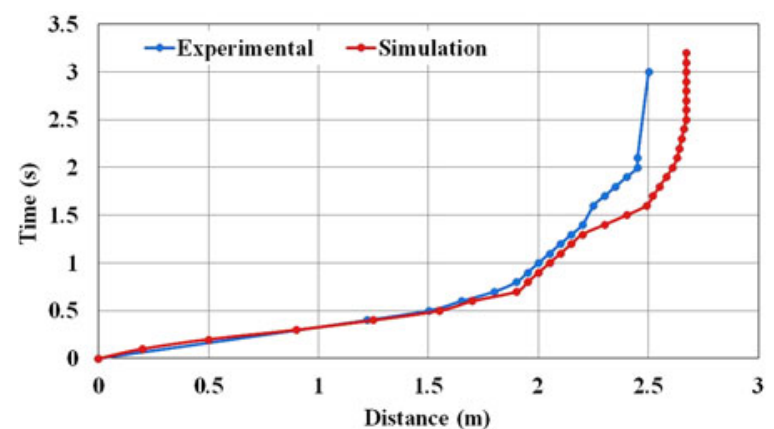

Fig. 11. Comparison in terms of position of leading edge with time (Case 1).

Table 1. The efficiency factor for the experimental and simulated results in the different cases

Area 1 Area 2 Efficiency factor (\%)

\begin{tabular}{lccc}
\hline Case 1 & & & \\
$\quad$ Experiment - weight of deposited sand (kg) & 0.45 & 1.1 & 76 \\
SPH - number of deposited particles & 2943 & 5736 & 72 \\
Case 2 & & & \\
$\quad$ Experiment - weight of deposited sand $(\mathrm{kg})$ & 0.06 & 0.92 & 48 \\
SPH - number of deposited particles & 0 & 5184 & 43 \\
Case 3 & & & \\
$\quad$ Experiment - weight of deposited sand (kg) & 0.38 & 1.25 & 80 \\
SPH - number of deposited particles & 2107 & 7054 & 76 \\
& & & \\
\hline
\end{tabular}

\subsubsection{Results and analysis}

Figure 13 presents a series of snapshots of the avalanche hitting the circular cylinder, showing the particles positions and free-surface shape together with the velocity field at different times. The result obtained by the SPH method is compared with the experimental images and the superimposed computed boundary obtained from the hydraulic avalanche model solution by Cui and others (2013). The time intervals between snapshots are equal to 0.72 non-dimensional units. Since the gate was not in the camera shots, the

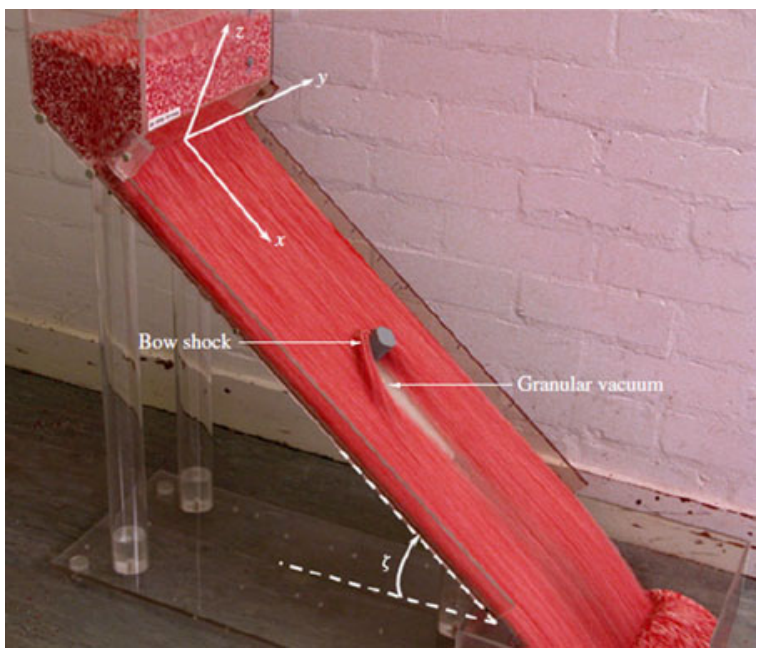

Fig. 12. Experimental setup showing the flow past a circular cylinder on a chute inclined at an angle $\zeta$ to the horizontal (Cui and others (2013)).
Table 2. The values of the soil parameters used to simulate the flow of granular avalanche around a circular cylinder

\begin{tabular}{ll}
\hline Parameter & Value \\
\hline Density $\left(\mathrm{kg} \mathrm{m}^{-3}\right)$ & $\rho=1500$ \\
Young's modulus $(\mathrm{MPa})$ & $E=150$ \\
Friction angle & $\phi=26^{\circ}$ \\
Poisson ratio & $v=0.3$
\end{tabular}

comparison of the simulation and the experiment starts from the distance $x=8$ units at time $t=7.4$ units.

When the particles are released from the gate, the flow front starts to propagate uniformly, and the flow was fast and thin. When the particles start to hit the cylinder, the flow continues to propagate downstream except for those particles that collide with the obstacle and are largely affected by the obstacle (Figs $13 \mathrm{~b}$ and f). A jump in the flow thickness and velocity, which is named as 'bow shock wave' starts to develop at the front of the cylinder by $t=8.84$ units (Figs $13 \mathrm{c}$ and g). The bow shock continues to grow slightly in height and to move upstream until the oblique shocks on either side of the obstacle are almost fully developed by $t=10.28$ (Figs 13d and h). By $t=12.44$ units, (Figs $13 \mathrm{e}$ and i), the flow reaches a steady-state regime with no further growth within the field of view, and the granular vacuum clearly appears downstream of the cylinder and forms a triangular shape.

The developing process of the bow shocks by the present computation is in a close agreement with the experimental results. For the vacuum boundary, the closed triangular region obtained by the simulation seemed to be larger than the one formed in the experiment. Generally, we can conclude that this model captures most of the essential aspects of the observed phenomena.

\subsection{Gravity granular flows past tetrahedral wedge}

\subsubsection{The model description}

The tetrahedral wedge was proposed to protect the Schneefernerhaus at Zugspitze, Germany, against a large avalanche with a 100 a recurrence equal to a snow layer of $8 \mathrm{~m}$ depth moving down the mountain (Tai and others, 1999). The Schneefernerhaus was an old hotel, which was renovated and transformed into a research laboratory for environmental and climatological research in 1999. It is situated on a mountain slope inclined by $\sim 45^{\circ}$ (Fig. 14a).

In a first study aimed at protecting this building against avalanche, a tetrahedral wedge was designed to divert the flow and guide the snow pass the building on either side. Tai and others (2002) decided to carry out laboratory experiments to simulate this event. Two models at scales 1 : 100 and $1: 300$ were used to perform their experiments. In our study, the two-scale experiments were simulated using the current SPH model. In the experiments, the mountain flank modeled as an inclined plane of a $45^{\circ}$ slope angle is made from Plexiglas. The models of Schneefernerhaus and of the wedge were cut from plastic and wood blocks, respectively.

Semolina flour grains, $0.8 \mathrm{~mm}$ in diameter, and plastic beads were used as the model of 'dry snow'. The internal 

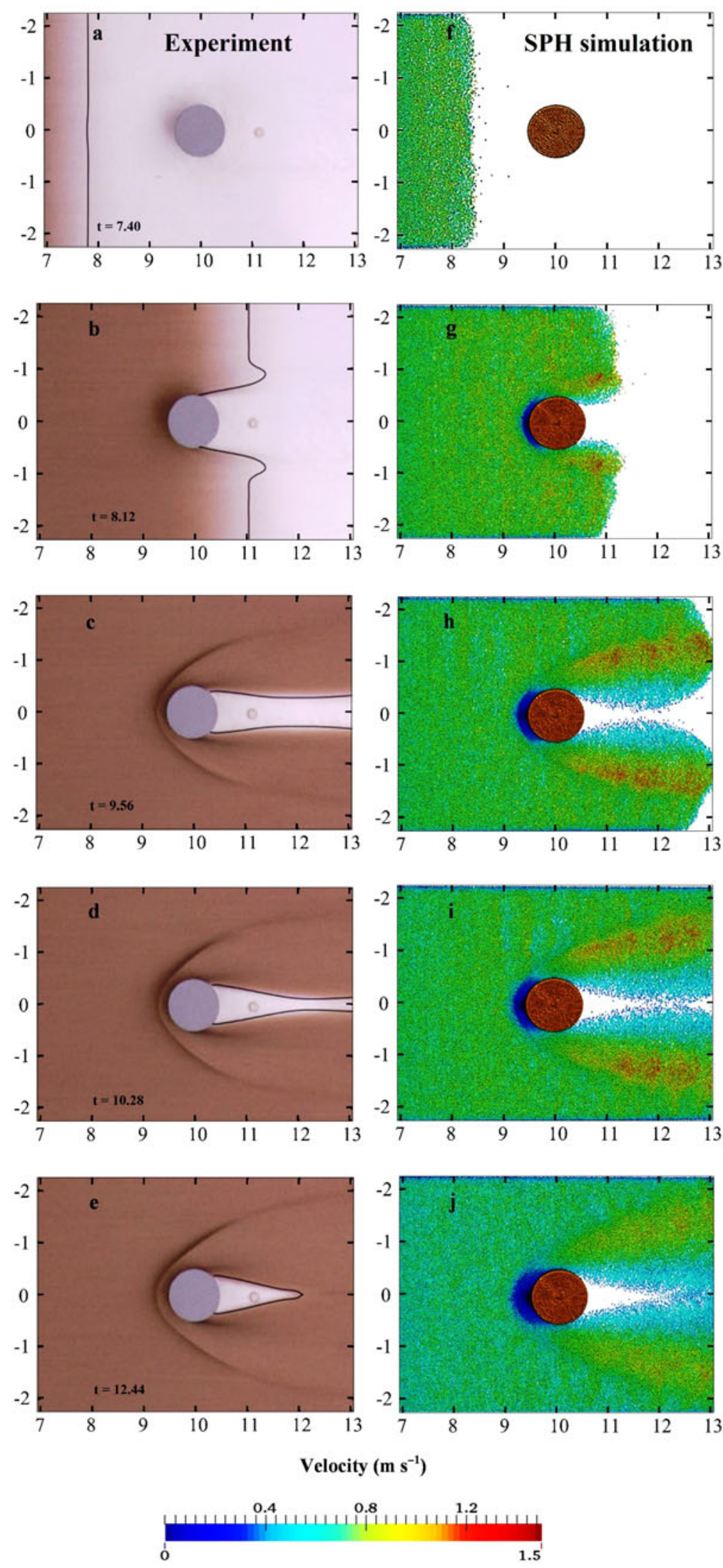

Fig. 13. Comparison between computation and experiment results at different time steps $(t=7.4,8.12,9.56,10.28$ and 12.44 dimensionless units) showing the continuation of the time-dependent development of a bow shock and a vacuum boundary.

angle of friction of these materials was within the range of the snow. The used materials were initially filled in a tank with a gate at the upstream end of the slope. Figure $14 \mathrm{~b}$ shows the experimental setup with scale $1: 300$.

\subsubsection{Numerical simulations}

The number of particles used to simulate the semolina and plastic beads masses was 165,000 , with $0.002 \mathrm{~m}$ initial distance between particles. The material constants used 

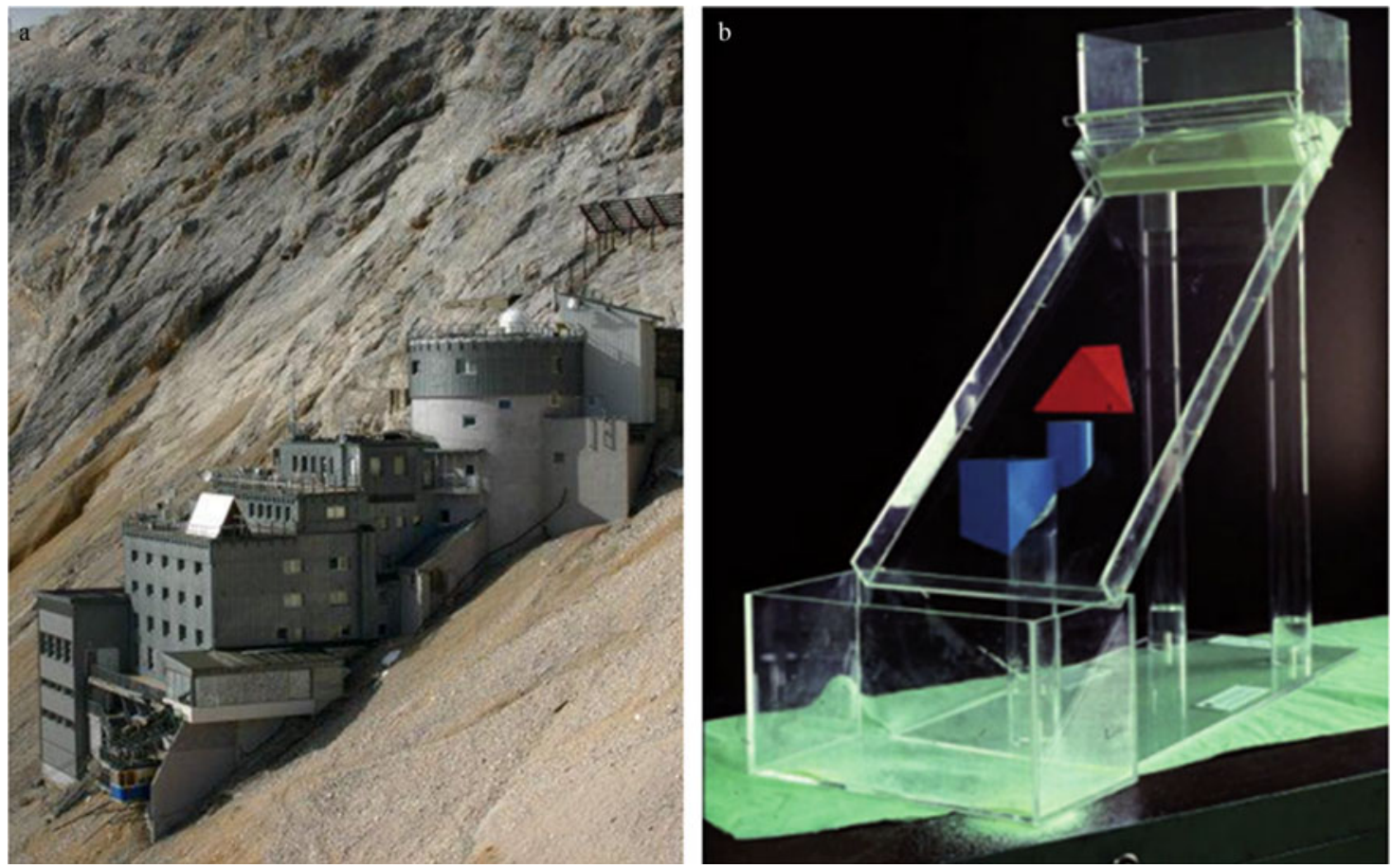

Fig. 14. (a) The Schneefernerhaus at the Zugspitze, Germany at $2700 \mathrm{~m}$ on a rather planar mountain slope inclined at $\sim 45^{\circ}$. (b) $\mathrm{A}$ model reproduction together with tetrahedral wedge type avalanche protection.

Table 3. The material parameters used to simulate the gravity granular flows past tetrahedral wedge

\begin{tabular}{lll}
\hline Parameter & \multicolumn{2}{c}{ Value } \\
\cline { 2 - 3 } & Semolina & Plastic beads \\
\hline Density $\left(\mathrm{kg} \mathrm{m}^{-3}\right)$ & $\rho=600$ & $\rho=890$ \\
Young's modulus (MPa) & $E=5000$ & $E=900$ \\
Friction angle & $\phi=35^{\circ}$ & $\phi=38^{\circ}$ \\
Poisson ratio & $v=0.49$ & $v=0.42$ \\
\hline
\end{tabular}

in the calculation for the two cases are illustrated in Table 3.

\subsubsection{Results and analysis}

Figure 15 shows three snapshots of the motion of a layer of semolina. This layer represents the motion of an $8 \mathrm{~m}$ layer down the slope, past and around the obstacle and the Schneefernerhaus building at three different stages compared with numerical results of the SPH simulations.

From the snapshots, we can see how the obstacle diverts the flow. A normal shock is formed at the pyramid top and extends on both flanks of the pyramid to form an oblique shock, which can be clearly seen from the streamlines in the photograph as well as the particles spreading in the numerical simulation. At the downstream region of the obstacle, the flow rapidly spreads in the transverse direction and forms an expansion fan far from the protected area, which is also well described by the SPH simulation.

Figure 16 shows a comparison of three different results. An experimental photograph (scale 1 : 100), the numerical results obtained by applying the Savage and Hutter (1989) theory using the two-dimensional (2-D) NOC scheme (Jiang and Tadmor, 1997), and the SPH simulation, representing the steady flow past the defense structure. The oblique shocks on both sides of the defense structure are seen from the streamlines in the experiment, computed flow thickness from the 2-D NOC scheme, and the particle distribution together with the velocity field. Also, the particle-free region was formed, which shows that the protected zone is well described in the $\mathrm{SPH}$ results.

From the above results, it is clear that the SPH method can well describe the properties of the flow around the tetrahedral obstacle and can quantitatively describe the protection region.

Figure 17 demonstrates a horizontal cross section of the flow depth along the line $x=5.6$ dimensionless units, which crosses the upper part of the pyramid at $t=10$ dimensionless units. The solid curve represents the Savage and Hutter (1989) theory solution utilizing the 2-D NOC scheme, and the particles distribution gives the cross-sectional shape obtained by the SPH simulation.

The shocks are visible on either side of the defense structure. Behind the shock, the avalanche thickness is approximately four times that of the Savage and Hutter theory solution, and three times that of the present SPH model as large as in front of the shock. In the two solutions, the shocks are symmetrical though they have different shapes.

\section{CONCLUSION}

A novel 3-D computational model based on the SPH method has been developed for simulating the granular flow past different types of obstacles. The elastic-perfectly plastic model has been implemented in the SPH framework to model the granular materials. The present model was validated by an experiment on the collapse of the 3-D axisymmetric column of sand. Good agreement was observed between the numerical and experimental results. 

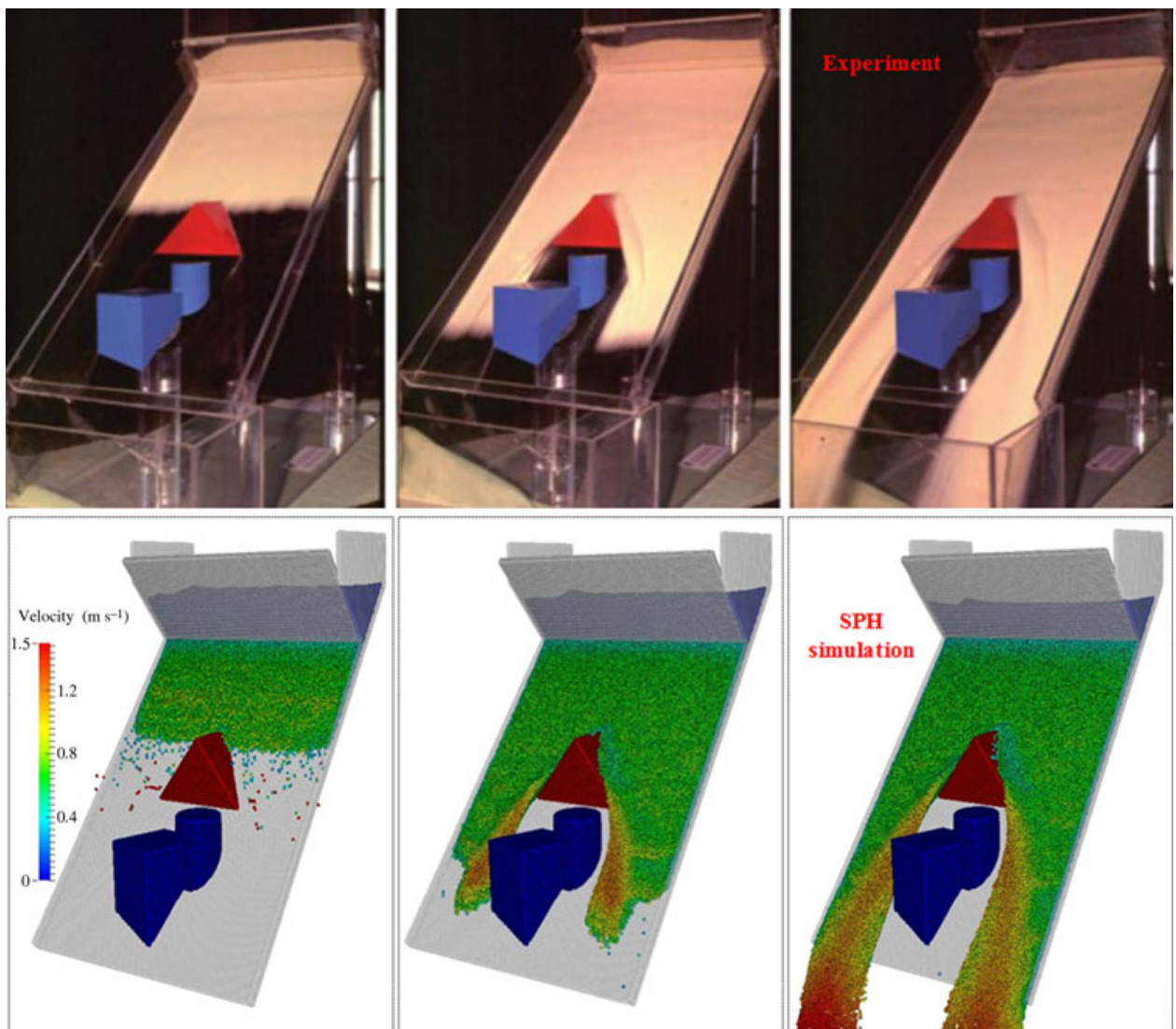

Fig. 15. Caparison between experimental and numerical results at three different stages showing the motion of a layer of semolina past and around the obstacle and the Schneefernerhaus building.
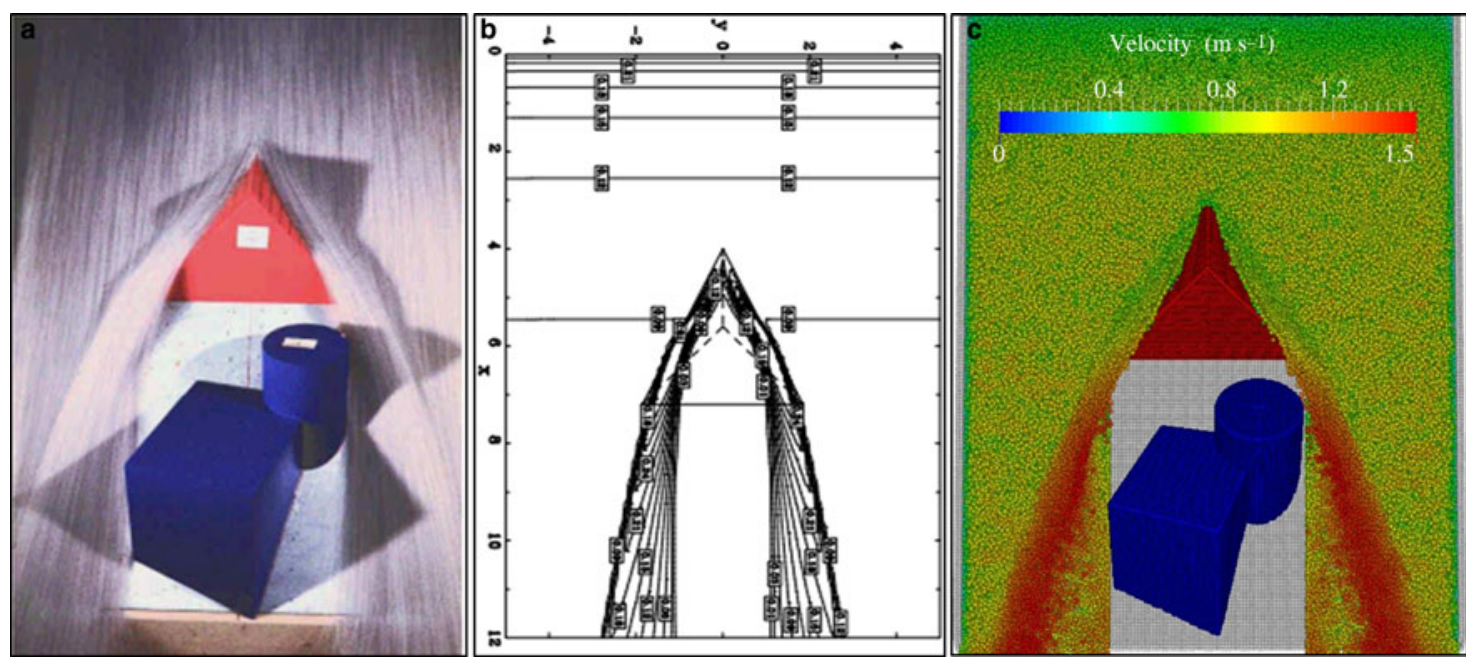

Fig. 16. Comparison between: (a) the experimental results (scale $1: 100$ ), (b) numerical results obtained from the 2-D NOC scheme and (c) SPH simulation representing the steady flow past the defense structure.

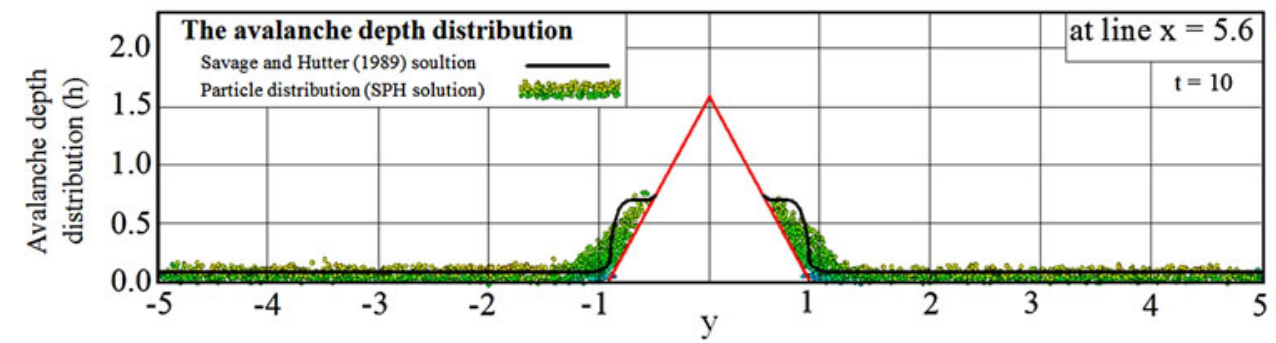

Fig. 17. Cross section of the avalanche depth distribution along the line $x=5.6$ through the top of the pyramid. 
Granular flow past three types of obstacles, a group of stake rows with different spacing, circular cylinders and tetrahedral wedge have been numerically simulated using the present SPH model. The computational results were compared with the experimental results and two existing numerical results to check the capabilities of the proposed model. The numerical results in the first case in terms of the final granular deposition shapes, spreading of the particles and the position of the leading edge are found to be in good agreements with the experimental results. Although the efficiency factor from the experimental results in all cases is slightly greater than the calculated values from the $\mathrm{SPH}$ results, the error is $\sim 5 \%$.

The simulation of granular free-surface flow around a circular cylinder, and a tetrahedral obstacle shows that the SPH method can capture and describe the formation of the bow shock, normal shock and oblique shock around the obstacle, and can describe the protected area as observed in the experiments, the hydraulic avalanche model solution and Savage and Hutter (1989) theory solution.

This study suggests that SPH could be a powerful method for solving problems dealing with granular materials subjected to large deformation and could be used to design real avalanche defenses.

\section{ACKNOWLEDGEMENT}

A. M. Abdelrazek would like to acknowledge the financial support from the Japanese government (MEXT), and JSPS KAKENHI Grant Number 15F15369.

\section{REFERENCES}

Abdelrazek AM, Kimura I and Shimizu Y (2014a) Comparison between SPH and MPS methods for numerical simulations of free surface flow problems. J. Jpn. Soc. Civ. Eng.., Ser. B1 (Hydraulic Engineering), 70(4), I_67-I_72 (doi: 10.2208/jscejhe. 70.I_67)

Abdelrazek AM, Kimura I and Shimizu Y (2014b) Numerical simulation of snow avalanches as a bingham fluid flow using $\mathrm{SPH}$ method. Proceedings of river flow 2014, 7th international conference on fluvial hydraulics, Lausanne, Switzerland, 3-5 September 2014, 1581-1587, CRC Press, Taylor \& Francis Group (doi: 10.1201/b17133-210)

Abdelrazek AM, Kimura I and Shimizu Y (2014c) Numerical simulation of a small-scale snow avalanche tests using non-Newtonian SPH model. J. Jpn. Soc. Civ. Eng., Ser. A2 (Applied Mechanics (AM)), 70(2), I_681-I_690 (doi: 10.2208/jscejam.70.I_681)

Amicarelli A and 6 others (2011) SPH truncation error in estimating a 3D derivative. Int. J. Numer. Methods Eng., 87, 677-700 (doi: 10.1002/nme.3131)

Armstrong BR, Williams K and Armstrong RL (1992) The Avalanche Book. Golden, Colorado. Fulcrum Publishing

Bui Ha H, Sako K and Fukagawa R (2007) Numerical simulation of soil-water interaction using smoothed particle hydrodynamics (SPH) method. J. Terramech., 44, 339-346 (doi: 10.1016/j. jterra.2007.10.003)

Bui Ha H, Fukagawa R, Sako K and Ohno S (2008) Lagrangian meshfree particles method (SPH) for large deformation and failure flows of geomaterial using elastic-plastic soil constitutive model. Int. J. Numer. Anal. Methods Geomech., 32(12), 15371570 (doi: 10.1002/nag.688)

Bui Ha H, Fukagawa R, Sako K and WELLS JC (2010) Slope stability analysis and discontinuous slope failure simulation by elastoplastic smoothed particle hydrodynamics (SPH). Géotechnique, 61(7), 565-574 (doi: 10.1680/geot.9.P.046)

Chen W and Qiu T (2012) Numerical simulations for large deformation of granular materials using smoothed particle hydrodynamics method. Int. J. Geomech., 12(2), 127-135 (doi: 10.1061/(ASCE)GM.1943-5622.0000149)

Cui X and Gray JMNT (2013) Gravity-driven granular free-surface flow around a circular cylinder. J. Fluid Mech., 720, 314-337 (doi: 10.1017/jfm.2013.42)

Cui X, Gray JMNT and Johannesson T (2007) Deflecting dams and the formation of oblique shocks in snow avalanches at Flateyri, Iceland. J. Geophys. Res. Earth Surf., 112, F04012 (doi: 10.1029/2006JF000712)

Cummins SJ and Rudman M (1999) An SPH projection method. J. Comput. Phys., 152, 584-607 (doi: 10.1006/jcph.1999.6246)

Dalrymple RA and Knio O (2001) SPH Modelling of Water Waves. Proc. Coast. Dyn., '01, 779-787, Sweden (doi: 10.1061/40566 (260)80)

Faug T, Beguin R and Benoit C (2009) Mean steady granular force on a wall over-flowed by free-surface gravity-driven dense flows. Phys. Rev. E, 80, 021305 (doi: 10.1103/PhysRevE.80.021305)

Gingold RA and Monaghan JJ (1977) Smoothed particle hydrodynamics: theory and application to nonspherical stars. Mon. Not. R. Astron. Soc., 181, 375-389 (doi: 10.1093/mnras/181.3.375)

Gray JMNT and Cui X (2007) Weak, strong and detached oblique shocks in gravity-driven granular free-surface flows. J. Fluid Mech., 579, 113-136 (doi: 10.1017/S0022112007004843)

Gray JMNT, Wieland M and Hutter K (1999) Gravity-driven free surface flow of cohesionless granular avalanches over complex basal topography. Proc. R. Soc. A, 455, 1841-1874 (doi: 10.1098/rspa.1999.0383)

Gray JMNT, Tai YC and Noelle S (2003) Shock waves, dead zones and particle-free regions in rapid granular free-surface flows. J. Fluid Mech., 491, 161-181 (doi: 10.1017/S0022112003005317)

Grigourian SS, Eglit ME and lakimov IL (1967) New statement and solution of the problem of the motion of snow avalanche. In Snow, Avalanches \& Glaciers. Tr. Vysokogornogo Geofizich. Inst., Russia, 12, 104-113 (In Russian)

Hakonardottir KM and Hogg A (2005) Oblique shocks in rapid granular flows. J. Phys. Fluids, 17(7), 077101 (doi: 10.1063/ 1.1950688)

Hanifa T, Agra B and Christian F (2013) Three-dimensional smoothed particle hydrodynamics simulation for liquid droplet with surface tension. ISCS Sel., Papers (doi: arXiv: 1309.3868v1)

Hauksson S, Pagliardi M, Barbolini M and Jóhannesson T (2007) Laboratory measurements of impact forces of supercritical granular flow against mast-like obstacles. Cold Reg. Sci. Technol., 49, 54-63 (doi: 10.1016/j.coldregions.2007.01.007)

Hopkins PF (2015) GIZMO: a new class of accurate, mesh-free hydrodynamic simulation methods. Mon. Not, R. Astron. Soc., 450, 53-110 (doi: 10.1093/mnras/stv195)

Iverson RM (1997) The physics of debris-flows. Rev. Geophys., 35, 245-296 (doi: 10.1029/97RG00426)

Jiang G and Tadmor E (1997) Non-oscillatory central schemes for multidimensional hyperbolic conservation laws. SIAM J. SCi. Comput., 19, 1892-1917 (doi: 10.1137/S106482759631041X)

Jóhannesson T, Gauer P, Issler D and Lied K (2009) The design of avalanche protection dams: recent practical and theoretical developments. European Commission, Directorate-General for Research. Publication EUR 23339 (doi: 10.2777/12871) ISBN 978-92-79-08885-8. ISSN 1018-5593

Johnson CG and Gray JMNT (2011) Granular jets and hydraulic jumps on an inclined plane. J. Fluid Mech., 675, 87-116 (doi: 10.1017/jfm.2011.2)

Kabir MA, Lovell MR and Higgs CF, III (2008) Utilizing the explicit finite element method for studying granular flows. Tribol. Lett., 29(2), 85-94 (doi: 10.1007/s11249-007-9285-y)

Koch T, Greve R and Hutter K (1994) Unconfined flow of granular avalanches along a partly curved surface. II. Experiments and Numerical Computations. Proc.: Math. Phys. Sci., 445(1924), 415-435 (doi: 10.1098/rspa.1994.0069)

Libersky LD and Petschek AG (1991) Smoothed particle hydrodynamics with strength of materials. In Proceedings of the Next 
Free Lagrange Conference, 395, Springer, New York, 248-257 (doi: 10.1007/3-540-54960-9_58)

Liu GR and Liu MB (2003) Smoothed particle hydrodynamics: a mesh-free particle method. World Scientific Publishing, Singapore

Liu GR and Liu MB (2010) Smoothed particle hydrodynamics (SPH): an overview and recent developments. Arch. Comput. Methods Eng., 17, 25-76 (doi: 10.1007/s11831-010-9040-7)

Lube G, Huppert HE, Sparks RSJ and Hallworth MA (2004) Axisymmetric collapses of granular columns. J. Fluid Mech., 508, 175-199 (doi: 10.1017/S0022112004009036)

Lucy L (1977) A numerical approach to testing the fission hypothesis. Astron. J., 82, 1013-1024 (doi: 10.1086/112164)

Mangeney-C A and 6 others (2003) Numerical modeling of avalanches based on Saint-Venant equations using a kinetic scheme. J. Geophys. Res, 108, 2527 (doi: 10.1029/2002JB002024)

Monaghan JJ (1994) Simulating free surface flows with SPH. J. Comput. Phys., 110, 399-406 (doi: 10.1006/jcph.1994.1034)

Monaghan JJ (2002) SPH compressible turbulence. Mon. Not. R. Astron. Soc., 335(3), 843-852 (doi: 10.1046/j.1365-8711.2002. \$05678.x)

Monaghan JJ and Kocharyan A (1995) SPH simulation of multiphase flow. Comput. Phys. Commun., 87, 225-235 (doi: 10.1016/ 0010-4655(94)00174-Z)

Monaghan JJ and Lattanzio JC (1985) A refined particle method for astrophysical problems. Astron. Astrophys., 149, 135-43

Savage SB (1979) Gravity flow of cohesionless granular materials in chutes and channels. J. Fluid Mech., 92, 53-96 (doi: 10.1017/ S0022112079000525)

Savage SB and Hutter K (1989) The motion of a finite mass of granular material down a rough incline. J. Fluid Mech., 199, 177-215 (doi: 10.1017/S0022112089000340)
Sigurdsson F, Tomasson GG and Sandersen F (1998) Avalanche defenses for Flateyri, Iceland. from hazard evaluation to construction of defences. (Technical Report 203). Norw. Geotech. Inst., Oslo

Silbert LE and 5 others (2001) Granular flow down an inclined plane: bagnold scaling and rheology. Phys. Rev. E, 64, 051302 (doi: 10.1103/PhysRevE.64.051302)

Sovilla B, Schaer M, Kern M and Bartelt p (2008) Impact pressures and flow regimes in dense snow avalanches observed at the Vall' ee de la Sionne test site. J. Geophys. Res., 113, F01010 (doi: 10.1029/2006JF000688)

Tai YC, Wang Y, Gray JMNT and Hutter K (1999) Methods of similitude in granular avalanche flows. Adv. Cold-Reg. Therm. Eng. Sci.-Lect. Notes Phys., 533, 415-428 (doi: 10.1007/ BFb0104200)

Tai YC, Gray JMNT, Hutter K and Noelle S (2001) Flow of dense avalanches past obstructions. Ann. Glaciol., 32(1), 281-284 (doi: 10.3189/172756401781819166)

Tai YC, Noelle S, Gray JMNT and Hutter K (2002) Shock capturing and front tracking methods for granular avalanches. J. Comput. Phys., 175, 269-301 (doi: 10.1006/jcph.2001.6946)

Vreman AW, Al-Tarazi M, Kuipers JAM, Van Sint Annaland M and Bokhove O (2007) Supercritical shallow granular flow through a contraction: experiment, theory and simulation. J. Fluid Mech., 578, 233-269 (doi: 10.1017/S0022112007005113)

Yaidel RL, Dirk R and Carlos RM (2013) Dynamic particle refinement in SPH: application to free surface flow and non-cohesive soil simulations. Comput. Mech., 51, 731-741 (doi: 10.1007/ s00466-012-0748-0)

Yellin K, Saito Y, Kimura I, Otsuki M and Shimizu Y (2013) Refinement of simulation model for practical design of energy dissipater for snow avalanche. JSSI \& JSSE Joint Conference on Snow and Ice Research 